\title{
Research on Solar Power Generation Control System Based on 360 Degree Rotate Double-Motor
}

\author{
Zheng WANG ${ }^{1}$ and Xuezeng JIA \\ Chongqing Chemical Industry Vocational College, Chongqing 401220, China
}

\begin{abstract}
Aiming at the problems of low utilization rate of solar energy and poor anti-interference ability of tracking structure solar energy control system in fixed structure solar energy device, this paper designs a dual axis high-precision solar tracking system based on four quadrant rule. The system adopts two ways: automatic tracking and manual correction. The system uses four photoresistors as detection elements, uses the four quadrant principle to judge the tracking offset angle, and drives two-dimensional two axis stepper motor through STC89C52 processor to achieve the purpose of vertical angle, so as to ensure that the solar panel is always in the state of maximum light receiving surface; When the system is disturbed, it can be judged according to the change of the photosensitive resistance in the energy monitoring system, and the artificial correction can be realized by modulating the size of the divider resistance, which can basically achieve $360^{\circ}$ Automatic rotation tracking. In addition, the energy monitoring system based on LabView is designed. Through the real system analysis, it can be concluded that the photoelectric energy conversion rate of the fixed solar device is increased by $32.4 \%$.
\end{abstract}

Keywords. Solar energy control system, 360 degree rotate double-motor, solar tracking system

\section{Introduction}

Over exploitation of energy leads to environmental pollution and the decrease of nonrenewable energy. Adhere to sustainable development, need to continue to increase the development and utilization of new energy [1]. As a kind of new energy, solar energy is renewable, pollution-free, inexhaustible and inexhaustible. It has become one of the important choices for people to deal with energy shortage, energy conservation and emission reduction. At present, solar energy is applied to various fields through heat collection, water supply, heating and power generation. However, due to the continuous change of solar radiation space and the interference of weather and other factors, the collection and utilization of solar energy is difficult [1-2], resulting in the low photoelectric conversion rate of solar energy.

Photovoltaic tracking technology is an effective way to solve the above problems [3]. Through the literature analysis, the common automatic tracking is divided into solar motion tracking and photoelectric tracking. The diurnal motion is controlled by

${ }^{1}$ Corresponding Author, Zheng WANG, Chongqing Chemical Industry Vocational College, Chongqing 401220, China; Email: 13752972040@163.com; 32136297@qq.com. 
time, longitude and reference longitude, which is also called time control system; Photoelectric tracking is controlled by four quadrant rules, also known as optical control system. Li [2] et al. Proposed a high-precision solar tracking system based on FPGA, in which the solar motion and photoelectric tracking methods are fully integrated. Compared with the non-tracking situation, the energy conversion rate is increased by 30\%; Zhang [3] et al. proposed the method of combining time control and light control to maximize the tracking target, and the experimental results are significantly higher than those of fixed structure; Ling [4] realized horizontal and vertical tracking control based on DSP, and did not conduct energy conversion evaluation. In this paper, a solar tracking system based on four quadrant rule is designed according to photoelectric automatic tracking mode and human correction mode. In addition, the solar energy monitoring system based on Labview is designed. By collecting the open circuit voltage and current of solar energy, the average power of the system and the relatively fixed solar energy system is calculated, and the utilization rate of solar energy is compared through the average power.

\section{Design of Solar Tracking Control System}

\subsection{Principle of Photoelectric Detection Sensor}

The system uses four photoresistors as photoelectric sensors. According to the fourquadrant rule, the solar panel is divided into four quadrants, and a photosensitive resistor is installed at the edge of each quadrant to receive the light from the East, West, North and south directions. The four photosensitive resistors form a symmetrical structure [4-5] with the center of the solar panel. The structure diagram is shown in figure 1.

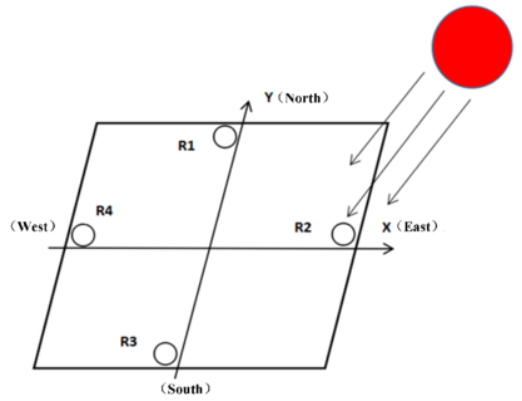

(a) Schematic diagram of oblique solar radiation

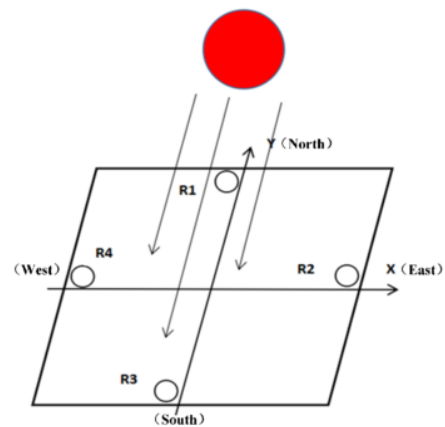

(b) Schematic diagram of direct solar radiation

Figure 1. Structural diagram of solar panel.

When the sun irradiates the solar panel, due to the different light intensity of each quadrant, the voltage on R1, R2, R3 and R4 is determined as U1, U2, U3 and U4 respectively by the acquisition and $\mathrm{AD}$ conversion circuit. As shown in figure $1 \mathrm{a}$, at the current positions $\mathrm{U} 2>\mathrm{U} 4$ and $\mathrm{U} 1>\mathrm{U} 3$, the motor on the $\mathrm{x}$-axis rotates to the East, and the motor on the $\mathrm{y}$-axis rotates to the North until $\mathrm{U} 1 \approx \mathrm{U} 3$ and $\mathrm{U} 2 \approx \mathrm{U} 4$ stop, and the solar panel tracks to the vertical position of sunlight. 


\subsection{Photoelectric Automatic Tracking Mode}

Due to the influence of weather changes, the solar panel cannot be guaranteed to have enough light. The literature basically uses the daily motion mode to control the motor tracking $[2-4,6]$. In this paper, by adjusting the size of the divider resistor in the detection circuit, changing the sensitivity, the detection and tracking under weak light is realized. Through many experiments, it is shown that the divider resistance ranges from 50 to $300 \Omega$. The modulation can meet the data acquisition of various light intensities.

\subsection{Control System}

The system structure is mainly composed of MCU control, photoelectric sensor, motor drive, wireless control, function key, acquisition and upper computer monitoring. The control system structure is shown in figure 2.

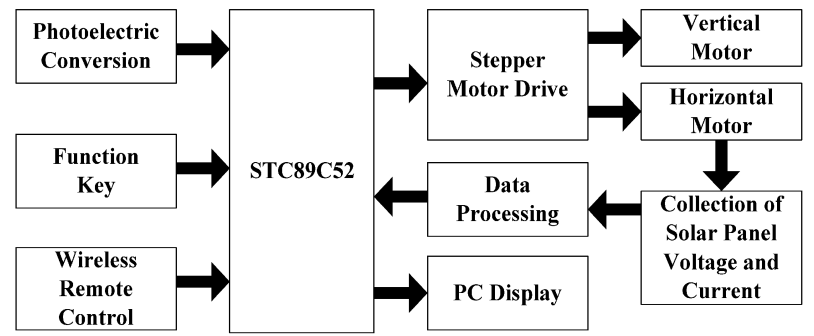

Figure 2. Structure diagram of control system.

The control part is the most important part, which is connected with other parts of the external circuit to receive and process the internal data, and then output it. This system uses 52 single-chip microcomputers of STC series. Compared with other types of single-chip microcomputer, it has the advantages of many types, fast execution speed and convenient operation. STC89C52 controls the angle of stepper motor by sending pulse signal to achieve the rotation of solar panel; The function key designed by inching button and the wireless remote control module designed by pt2272-m4 chip are used to realize the independent forward and reverse control of two stepper motors and the manual tracking correction function. The AD conversion module of single chip microcomputer is used to collect the voltage and current, realize the energy collection and provide the upper computer analysis; The data processing and display module designed by Labview is used to realize real-time display of voltage and current and comparative analysis of energy.

Stepper motor is used as tracking actuator and digital pulse is used as signal drive. A driver is needed to realize the conversion and distribution of digital pulse. The system is realized by ULN2003 chip, and the driving circuit is shown in figure 3. But in the process of experiment, the driver is affected by the jitter near the natural frequency point, which leads to the rapid opening and closing of the relay. Through the analysis, the jitter elimination algorithm is embedded in the program to realize the normal switching of the relay. 


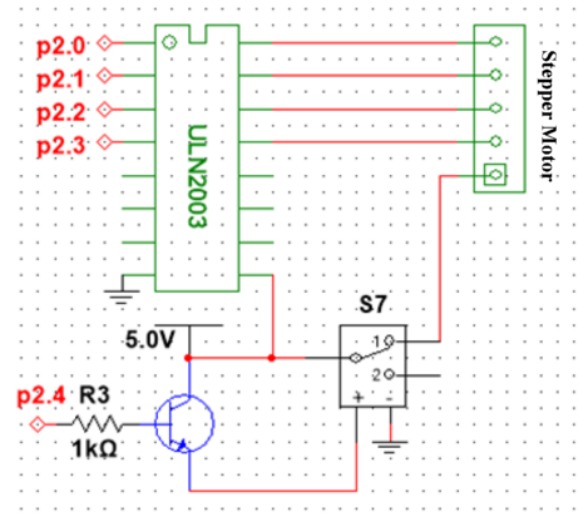

Figure 3. Stepping motor drive circuit.

\section{Mechanical Design of the System}

The bracket used in this system is X-Y double axis design, which can realize 360 degree positioning according to different directions of the sun ${ }^{\circ}$ we're tracking. One motor drives the other motor to rotate horizontally, and the other motor drives the solar panel to rotate vertically [7]. The structure diagram is shown in figure 4.

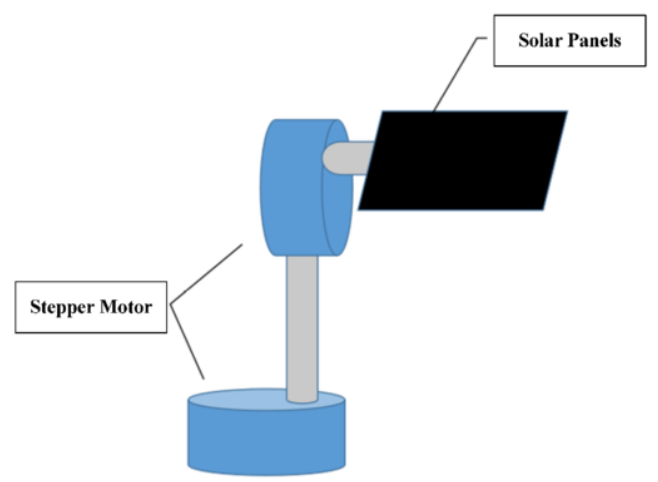

Figure 4. Solar mechanical structure.

\section{System Main Program Design}

The functions of the whole system include solar orientation detection, key control, wireless control and motor drive. The software scheme is that the system initializes the program after startup, and then enters the tracking program after initialization; Then the collection circuit collects the current and voltage generated by the solar panel, and sends it to the host computer for data processing and real-time detection. At the same time, the rotation of the motor can be controlled manually by the button or wireless control. The system operation flow chart is shown in figure 5 . 


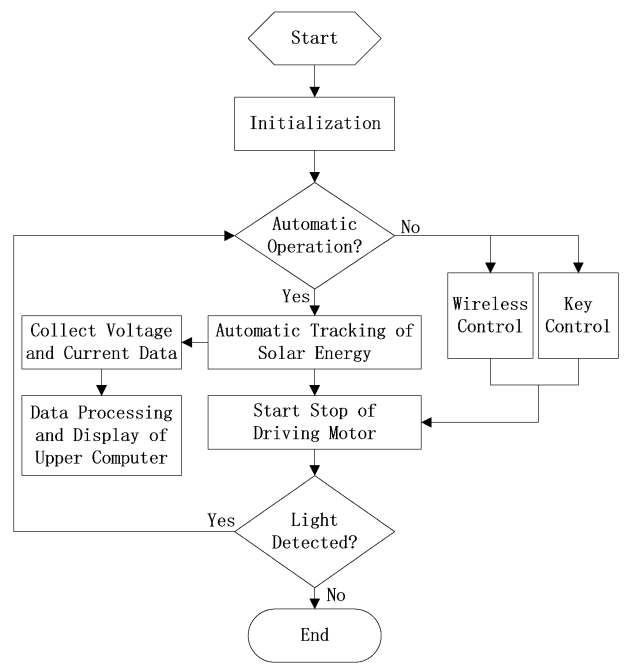

Figure 5. System operation flow chart.

\section{Design of Energy Detection System}

In order to better compare the power generation of fixed solar panels and hybrid tracking system solar panels, Labview is used to design an energy monitoring system. The system uses image display, average power calculation and gain comparison methods to record the power generation of solar panels more accurately, and gives a scientific evaluation standard.

\subsection{Monitoring System}

LabVIEW is one of the most widely used data acquisition and development environments in the world. It is mainly used in data acquisition, data analysis, data display, instrument control and other fields, and is suitable for a variety of different operating system platforms. Compared with the traditional programming language, Labview adopts powerful graphical language ( $G$ language) programming, which is oriented to test engineers rather than professional programmers. The human-computer interaction interface is intuitive and friendly, and has strong data visualization analysis and instrument control ability [8].

The interface uses the waveform diagram to realize the real-time data display, uses the array index function to decompose the array data of voltage and current transmitted by the lower computer, calculates the average power and relative gain of the system through basic calculation, and finally stores the voltage, current and average power in the database through labsql sub VI module to realize data storage and analysis. The solar energy monitoring system based on Labview is shown in figure 6 . 


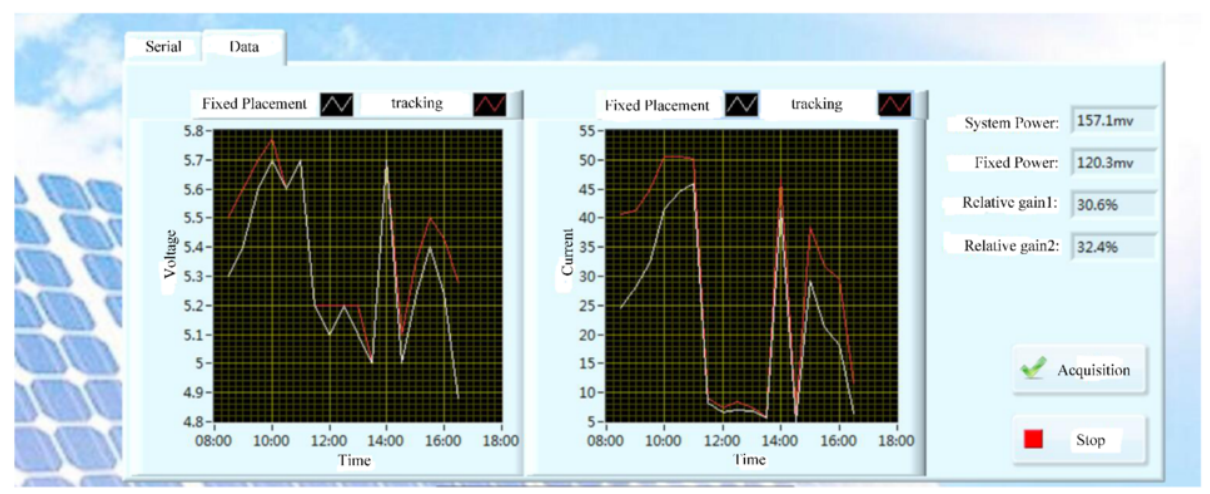

Figure 6. Energy monitoring system.

\subsection{Communication of Upper Computer}

The output voltage and current of the solar panel are collected into the MCU through the ad module and sent to the upper computer energy monitoring system through the serial port. The upper computer realizes the serial communication function through the visa sub VI module. The program diagram of serial communication setting is shown in figure 7 .

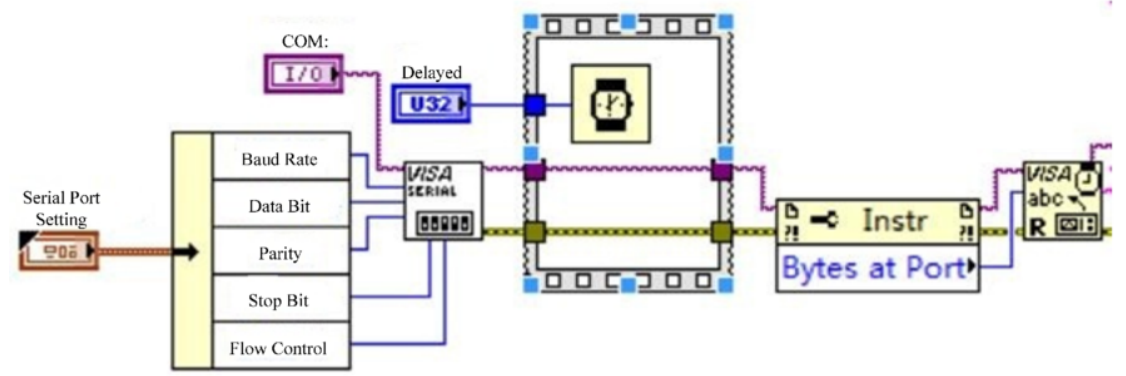

Figure 7. Serial communication settings.

\section{Experimental Results and Analysis}

\subsection{Solar Panel Parameters}

The system adopts Risym 5V polycrystalline solar panel, and the relevant parameters are shown in table 1.

Table 1. Solar panel parameters.

\begin{tabular}{ll}
\hline Maximum Power & $150 \mathrm{~mW}$ \\
\hline Working Voltage & $5 \mathrm{~V}$ \\
\hline Working Current & $0 \sim 30 \mathrm{~mA}$ (Depending on Light Intensity) \\
\hline Size & $53 * 30 \mathrm{~mm}$ \\
\hline Test Conditions & $\mathrm{AM} 1.5,25^{\circ} \mathrm{C}, 1000 \mathrm{w} / \mathrm{M}^{2}$ \\
\hline
\end{tabular}




\subsection{Experimental Results and Analysis}

The power of solar panels is calculated by testing the open circuit voltage and short circuit current of solar panels. At the same time, the fixed mode and tracking mode are used to achieve energy collection. Through the comparison of power generation, the comparison chart of power generation of the two modes can be obtained, as shown in figure 8 .

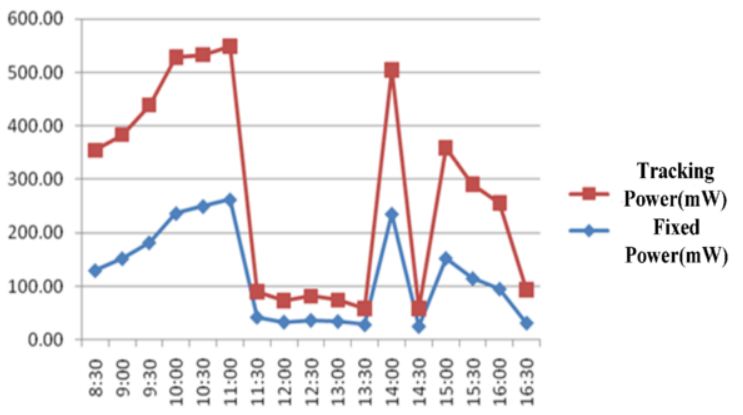

Figure 8. Comparison of power generation of two modes.

The weather conditions tested are: Sunny in the morning, less cloudy; It was cloudy at noon; It's cloudy in the afternoon. Data is collected every half an hour. It can be seen from the figure that it is sunny between 8:30 and 11:30 in the morning, and the power of the tracking system is significantly higher than that of the fixed tracking system. After 11:00, the main reason for the change of power is that the clouds block the sun. At this time, the power of day tracking or adjustable voltage divider resistance acquisition is not significantly higher than that of fixed one. According to the analysis of the solar energy monitoring system based on Labview, the average power of the system from 8:30 to $16: 30$ is $159.1 \mathrm{mw}$, the average power of the fixed solar panel is $120.3 \mathrm{mw}$, and the power gain is $30.6 \%$; Excluding the power calculation from 11:00 to $13: 30$, the power gain is $32.4 \%$.

\section{Conclusions}

Aiming at the low utilization of solar energy, this paper analyzes the common tracking modes, and proposes a dual axis high-precision solar tracking system based on four quadrant rules. The system integrates the manual correction method of automatic tracking and partial resistance adjustment, and realizes $360^{\circ}$ High precision tracking of the system. In addition, through the upper computer monitoring system accurate analysis of the average power generated by solar energy, the results show that: tracking without light can only increase the power consumption of the system, cannot effectively improve the utilization of solar energy. In addition, the system has low cost and high practicability. 


\section{References}

[1] Liu L 2013 Design and Research of Solar Panel Automatic Tracking System (Heilongjiang University) pp 1-2.

[2] Li H and Wang R L 2016 Design of high precision solar tracking control system Power Technology (2) 388-389.

[3] Zhang S H, Wen X L, Shao P C, et al. 2018 Design of high precision two axis solar tracking control system Journal of Wuhan University of Technology 40 (3) 315-319.

[4] Ling J H 2017 Research and design of solar tracking system Information Technology and Informatization (16) 141-145.

[5] Wang J P, Wang J, Feng W, et al. 2015 Development and application of trough solar tracking control system Journal of agricultural engineering 31 (2) 45-50.

[6] Wang W L, Song J, Xie Y X, et al. 2017 Design of high precision all weather solar automatic tracking system Instrument Technology and Sensor (7) 76-78.

[7] Yao X Y, Zhang H M and Zhang X L 2012 Design of solar tracking system based on STM32 World Science and Technology Research and Development (4) 630-632.

[8] Yang Z R, Rao C, Zou J, et al. 2004 Data acquisition system based on LabVIEW Journal of Chongqing University 27 (2) 32-35. 